Dossier Le naturel et la qualité

\title{
Classer et nommer les fruits du châtaignier ou la construction d'un lien à la nature
}

\author{
Lucie Dupré \\ Anthropologue, INRA, MONA, 65 avenue de Brandebourg, 94205 Ivry-sur-Seine cedex, France
}

\begin{abstract}
La dualité qui colle depuis l'origine au produit du châtaignier, du marron à la châtaigne, permet d'illustrer l'enjeu que représente le fait de nommer et classer les objets qui nous entourent. Le point de vue de l'ethnologue a sa place dans ce dossier, car il montre que dans les processus socio-techniques qui organisent la transformation et la commercialisation d'un produit alimentaire, ce dont les autres articles rendent compte, les mots ont leur importance dans l'instauration d'un ordre parmi les choses. Comme on peut le voir dans le va-et-vient historique du marron et de la châtaigne, l'action de classer fonde et justifie de nouveaux échanges marchands.
\end{abstract}

La Rédaction

\section{Mots-clés :}

Ardèche ;

diversité variétale ; châtaigneraie ; environnement ; châtaigne ; marron glacé

\section{Keywords:}

Ardèche;

chesnut;

chesnut tree; sugared chesnut; biodiversity; environment

\begin{abstract}
Résumé - En français, il existe deux termes pour désigner les fruits du châtaignier (Castanea sativa) : «châtaignes » et «marrons ». Des différences existent entre ces deux catégories de fruits et la distinction ne relève pas d'un simple jeu de langage. Une approche ethnobotanique et socio-technique de la question variétale saisie dans son ampleur historique (depuis le XVIII e siècle) montre que tout ce qui ne fait pas marron, c'est-à-dire les variétés qui ne réunissent pas les exigences très sévères de la confiserie industrielle naissante à la fin du XIXe siècle, est déclassé dans la catégorie «châtaigne ». C'est sur cette tension entre la nature et la culture, entre la nourriture quotidienne et le mets festif, entre le tout juste comestible et l'excellence gastronomique que cet article propose de revenir. Cette tension est aujourd'hui retravaillée dans le processus de relance de la production, mais au bénéfice sémantique de la châtaigne comme en témoigne l'usage de plus en plus fréquent de ce mot récemment réhabilité.
\end{abstract}

\begin{abstract}
Categorization and naming of Sweet Chestnut fruit: constructing a link with nature. In French the fruit of Castanea sativa, the chestnut tree, are called either chattaignes or marrons. These names correspond to two different socio-technical and socio-political "objects". The first clearly refers to the tree it grows on and is associated with poverty, rough food, labour and living conditions in deprived areas, as abundantly stated in social and agronomic literature. The second appellation on the contrary evokes no link with the tree: the fruit's origin is concealed so that it may become eatable. It is associated with sugared chestnuts (marrons glacés), a highly refined food which only the affluent can buy. The first part of the paper analyses the construction of these two "objects" since the XVIIIth century through an ethnobotanical and historical approach to the classification of the many chestnut varieties. It focuses on the industrialisation of the processing of sugared chestnuts which developed in the late XIXth century in a nowadays renowned chestnut-producing French department. We show how those categories of fruit that, for technical reasons, cannot be processed into sugared chestnuts retain the appellation of châtaignes and have a less prestigious fate, with some of them even totally disqualified. The second part deals with the revival of chestnut production and lays emphasis on the present-day use of the word châtaigne, which gives visibility to what used to be considered disreputable in past centuries, a crop closely linked to wild nature. This is symptomatic of the growing consideration for nature, food quality and high-value landscapes that have been developed since the 1980s.
\end{abstract}

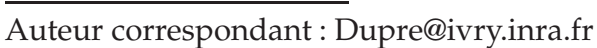


Les fruits gardent généralement le même nom de l'arbre à l'assiette. Le châtaignier (Castanea sativa Miller) fait exception : ses fruits sont connus sous deux noms différents : la châtaigne et le marron. Pour simplifier, disons que sur l'arbre, les fruits sont des châtaignes; ils deviennent des marrons dans l'assiette. Cette rupture sémantique est suffisamment rare pour qu'on s'y arrête un instant. Notre hypothèse est qu'elle permet de marquer une rupture avec un arbre, le châtaignier, qui renvoie à des territoires de moyenne montagne où la nature presque sauvage est potentiellement hostile et dont les populations sont laborieuses, sinon nécessiteuses. Dans la première partie de ce texte, nous analysons les modalités de cet arrachement à la nature dont la fabrication de l'objet marron résulte. Cette analyse s'appuie sur un corpus de différentes listes et nomenclatures issues de la littérature botanique et agronomique savante, datant essentiellement des XVIII ${ }^{\mathrm{e}}$ et XIX ${ }^{\mathrm{e}}$ siècles. C'est en effet à ce moment-là, et en Ardèche, que l'industrialisation de la fabrication du marron confit durcit encore davantage cette distinction, en l'adossant à ce qu'on peut appeler l'épreuve industrielle, sur laquelle nous revenons dans la seconde partie. Depuis une vingtaine d'années, le terme " châtaigne » tend à sortir de la disgrâce dans laquelle il était tenu. Ce phénomène est lié aux dynamiques de relance de produits dits traditionnels, dont la castanéiculture est l'objet (Dupré, 2002). Dans la troisième partie, nous examinons comment la réhabilitation du terme - et donc de l'objet - « châtaigne » renvoie à des préoccupations majeures qui structurent les pratiques contemporaines liées à la consommation alimentaire : l'environnement et la santé.

\section{Les classifications agronomiques et botaniques}

L'analyse développée dans cette partie s'appuie sur un corpus de catalogues de pépiniéristes, de dictionnaires généralistes ou spécialisés, d'inventaires monographiques, de traités et autres manuels qui décrivent et présentent le châtaignier et les nombreuses variétés de fruits depuis le XVIII ${ }^{\mathrm{e}}$ siècle. Lorsque le châtaignier est greffé, il se décline en un grand nombre de variétés qui ont donné lieu à une activité classificatoire soutenue. L'opposition canonique sauvage/domestique est la formule initiale et durable - quoique imparfaite - pour mettre de l'ordre dans la diversité végétale que le XVIII ${ }^{\mathrm{e}}$ siècle, saisi d'une "fureur» descriptive et classificatoire, va démêler fil à fil (Marchenay, 1981). Quelles sont les propriétés du sauvage et du cultivé? Quels sens sont mobilisés dans l'expertise? C'est ce que nous allons maintenant examiner en insistant sur les catégories les plus contrastées.

\section{Du sauvage au domestique : l'arbre et le fruit}

Au XVIII ${ }^{e}$ siècle, les définitions restent vagues et les critères retenus pour distinguer la châtaigne du marron mobilisent au moins deux sens : la vue (aspects extérieur et intérieur) et parfois le goût. Les trois grandes figures de la science agricole des XVIII ${ }^{\mathrm{e}}$ et $\mathrm{XIX}^{\mathrm{e}}$ siècles que sont Duhamel du Monceau, l'abbé Rozier et Adrien de Gasparin ne cachent ni l'ampleur de la tâche à accomplir, ni les difficultés à réduire la grande diversité des cultivars, souvent appelés «sortes » ou « espèces » et qui s'échelonnent entre le sauvage et le domestique.

En 1828, Louis Clerc, dans son Manuel de l'amateur de marrons et de châtaignes. . ., part de la «Châtaigne des bois » pour arriver à la «Châtaigne ordinaire » puis à la "Châtaigne printanière » qui est, dit-il, « la première en état d'être mangée ». Il énumère ensuite une théorie de variétés jusqu'au «vrai marron [...] presque rond, sans aucun zeste dans sa chair [et] sans contredit le meilleur de tous ». Les premières châtaignes sont sombres et velues, avec une peau intérieure pénétrante et une écorce épaisse. Elles sont à la limite de la comestibilité. Le marron, quant à lui, est un fruit sucré, fin, sans pénétration intérieure de peau, de couleur claire, à l'écorce lisse et fine. La corrélation entre la petite taille du fruit et le mauvais goût, voire la toxicité, est systématique (Clerc, 1828). Une telle lecture perdure un siècle plus tard. Henri Blin parcourt à son tour ce qui sépare la «châtaigne des bois » des « vrais marrons », en passant par la " châtaigne ordinaire » ou la «châtaigne commune » (Blin, 1904). Toutefois, il ne renonce pas à stabiliser une raison naturaliste purifiée des déterminations culturelles : lorsqu'il distingue les deux «sortes » de fruits, l'un (le marron) «de forme plus ronde [...] plus parfumé et plus savoureux » que l'autre (la châtaigne), il pointe les limites de l'exercice classificatoire des variétés lorsque celui-ci est effectué à partir des seuls fruits. Il plaide alors pour un nécessaire « retour à l'arbre » pour compléter l'expertise.

Depuis le XIX ${ }^{\mathrm{e}}$ siècle, l'arbre est couramment classé soit comme «châtaignier-proprement dit», soit comme « châtaignier-marronnier ». On ne s'étonnera pas d'apprendre que le premier est rustique, très productif en fruits de qualité médiocre, alors que le second est présenté comme un arbre fragile, de production relativement faible mais de qualité. À la nature généreuse qui assure à manger à tous s'oppose la culture plus sélective, mais moins prolifique, qui offre une qualité supérieure. L'ensemble de ces critères de classification de l'arbre et des fruits a traversé, de façon étonnante, la littérature savante ou grand public ${ }^{1}$.

\footnotetext{
1 Le Petit Larousse de 1995 précise que le «marronnier» est un «châtaignier d'une variété cultivée qui produit le marron Castanea ». Ce n'est qu'ensuite que ce dictionnaire évoque le marronnier d'Inde, espèce dont les fruits sont impropres à la consommation.
} 


\section{La disqualification de la châtaigne} et du châtaignier

On le pressent, l'opposition entre la châtaigne et le marron renvoie à deux mondes contrastés que Pierre Larousse résume de façon éloquente dans son Grand dictionnaire universel du XIX ${ }^{e}$ siècle, à l'article "châtaigne » (Larousse, 1866-1879) : «Les bonnes variétés sont admises sur les meilleures tables. Ce fruit forme presque à lui seul toute la nourriture des montagnes de l'Auvergne, des Cévennes, de la Corse et de plusieurs autres contrées. »

La disjonction entre l'accès aux « meilleures tables » et une consommation courante, sinon quotidienne, dans les régions productrices se fait sans le recours explicite à l'alternative châtaigne/marron qu'elle suppose : deux classes d'objets pour deux classes sociales. La première, élite gastronome et savante, est aussi urbaine et aisée que la seconde, populaire sinon nécessiteuse, est rurale et vit dans une dangereuse symbiose avec l'arbre, qui plus est dans des territoires marginaux. Les pentes des territoires castanéicoles sont, en effet, des zones intermédiaires entre l'ager et la silva. Elles s'intercalent entre l'espace cultural proprement dit et la montagne, espace sauvage dévolu à l'éleveur et au chasseur, inquiétantes figures des communautés rurales.

C'est une chose d'évoquer le châtaignier comme « un arbre de première nécessité qui sert aux habitants des campagnes et à la classe indigente d'unique nourriture ", comme le fait Louis Clerc en 1828; c'en est une autre d'en déduire, pour le condamner, l'état moral des populations qui en vivent, c'est-à-dire qui le produisent et s'en nourrissent. Depuis Adrien de Gasparin, qui, au milieu du XIX siècle, avançait qu'une "population alimentée presque uniquement par les fruits d'un arbre est nécessairement dans un état stationnaire ", jusqu'à Edmond Demolins, qui, au début du $X X^{\mathrm{e}}$ siècle, reprend à son compte l'argument - «L'exploitation du châtaignier ne favorise pas l'Expansion de la race parce qu'elle ne développe pas dans la population, ni les aptitudes agricoles, ni les aptitudes industrielles, ni les aptitudes commerciales » (Demolins, 1900) - , les " gens de châtaignes » sont présentés de façon récurrente comme des paresseux incapables de pratiquer une véritable agriculture moderne. En un mot, ce sont des sauvages vivant dans une proximité condamnable avec la nature. Et pourtant... La production a donné lieu, dès le XVIII e siècle, à une économie marchande dynamique et originale d'où est sorti. . . le marron. C'est, en effet, précisément à l'époque de Demolins, et en Ardèche, que la châtaigne s'ouvre au destin le plus glorieux et à l'épreuve la plus exigeante : la confiserie industrielle. Ce type de valorisation de certains fruits - on verra lesquels - accentue et entérine la disjonction entre sauvage/cultivé et châtaigne/marron. Mais l'Ardèche n'est pas pareillement concernée, puisque l'approvisionnement des usines ardéchoises et des centres urbains de la vallée du Rhône se fait principalement autour d'Aubenas. On s'éloigne des pentes de l'intérieur du département. .

\section{Le marron : une châtaigne sortie d'usine}

Depuis le $\mathrm{XVI}^{\mathrm{e}}$ siècle, $\mathrm{l}^{\prime}$ Ardèche est un département très actif dans la commercialisation des châtaignes. Certaines variétés ont acquis une réputation nationale et se font connaître sous l'appellation "marron ». Qu'est-ce que le «bon marron»? La réponse est simple : le marron est la meilleure des châtaignes; il se mange glacé ou confit; vient ensuite la bonne châtaigne, dite fine, qui se consomme fraîche (grillée ou bouillie); enfin, les autres fruits qui se mangent séchés, quand ils ne sont pas destinés au seul bétail. Le partage trifide déjà rencontré dans les classifications des variétés et des arbres (nature/culture, avec une catégorie intermédiaire) se déplace et se reporte au niveau du produit marchand. Il est, une fois de plus, particulièrement stable.

\section{Le « marron de Lyon » est ... a ardéchois}

Au XVII siècle, le «marron de Lyon » est probablement la plus ancienne catégorie commerciale connue. La question de sa réalité variétale a nourri un débat contradictoire dont Olivier de Serres s'est emparé en y reconnaissant la variété ardéchoise Sardonne, ancêtre de la future Bouche-Rouge. Jacky Reyne estime que la Sardonne ne devient un marron qu'après son passage par Lyon, où s'opère une traduction décisive de son nom local - et variétal - en appellation commerciale ${ }^{2}$ (Reyne, 1995). Le marron est donc avant tout un standard, c'est-à-dire une «forme réussie » résultant d'une "épreuve majeure » (Bessy et Chateauraynaud, 1995) - la circulation de la châtaigne dans l'espace marchand depuis l'essor commercial $\mathrm{du} \mathrm{XVI}^{\mathrm{e}}$ siècle. En quittant son lieu de production, le fruit change d'appellation, de statut et d'histoire. Si la place commerciale a été l'un des lieux propices à ce changement de nom, l'usine de confiserie en a été un autre. En 1951, des congressistes invités chez Clément Faugier assistent à la succession des «diverses opérations depuis l'arrivée des châtaignes jusqu'à leur sortie sous forme de marrons glacés ou de confiture » (Commission du châtaignier, 1951). C'est à l'appui d'un texte quelque peu antérieur, daté de 1935 et dont l'auteur est Charles Faugier, le petit-fils de Clément Faugier, que nous allons examiner en quoi consiste l'épreuve industrielle qui fait qu'une châtaigne devient un marron (Faugier, 1935).

\footnotetext{
${ }^{2}$ C'est là, du reste, une pratique extrêmement courante. Voir, par exemple, le jambon de Bayonne ou le brie de Meaux.
} 


\section{L'usine comme lieu de normativité ${ }^{3}$ et d'arrachement à la nature}

La toute première exigence est de disposer d'un fruit entier, c'est-à-dire non cloisonné. Le calibre important est également requis, puisque les marrons sont traités (et parfois même vendus) à l'unité. Mais, pour être nécessaire, cette seconde condition n'est pas encore suffisante. En effet, le «bon fruit à confire », précise encore l'auteur, doit être de belle forme, de bonne tenue pour ne pas s'effriter à la cuisson. L'épluchure intérieure (la seconde peau, plus fine, appelée le tan) doit encore s'enlever facilement, être exempte de rides pénétrant la chair - ce qui serait une cause de brisures. Mais ce n'est pas tout : d'autres critères apparaissent tout aussi décisifs, qui tiennent cette fois-ci à l'approvisionnement de l'usine en matière première, autrement dit à la production castanéicole.

Les industriels doivent pouvoir s'affranchir des aléas de la production (qualité, volume et régularité des récoltes) et de la commercialisation (homogénéité des lots, étiquetage précis, calibrage irréprochable). Les deux variétés ardéchoises, la fameuse Sardonne et la BoucheRouge, sont recalées. La première, ancienne reine et ambassadrice du marron glacé qui a régalé la cour de Louis XIV bien avant que sa fabrication ne soit industrialisée, a certes toutes les qualités requises, mais elle n'est pas produite en quantité suffisante pour permettre aux industriels d'organiser leur campagne. Quant à la Bouche-Rouge, aujourd'hui noble fruit de table, elle ne résiste pas à la cuisson et occasionne trop de pertes. De plus, elle s'épluche difficilement et sa forme parfois aplatie ne convient pas. Lorsque l'industrie se développe, l'entreprise s'oriente vers l'Italie, fournisseur principal de Clément Faugier depuis les années 1930. La crème de marron est apparue trois ans après l'ouverture de l'usine : elle était destinée à valoriser les fruits brisés en cours de confiserie. Aujourd'hui, elle est faite avec les très petites variétés de châtaignes identifiées sous divers noms péjoratifs : «la châtaigne d'industrie », « les communes » ou encore «le tout-venant». Nous y reviendrons.

L'industrie du marron glacé a fourni un repère d'excellence et a normalisé l'objet marron. Le corps scientifique s'est largement inspiré de la grille de sélection en fixant notamment un taux de cloisonnement de l'amande. Si les fruits sont cloisonnés à plus de 12 \%, la variété est dite "à caractère châtaigne »; sinon, on parle de fruits «à caractère marron ». L'aptitude à l'épluchage mécanique, la bonne tenue à la cuisson, le calibre et la forme arrondie font également partie de la grille de sélection des programmes d'amélioration variétale impulsés dès les années 1960 par l'Inra. Les variétés hybrides interspécifiques créées font partie de ces fruits «à caractère marron » dont il va être bientôt question. Mais, paradoxalement, les consommateurs - c'est du moins ce qui ressort

\footnotetext{
${ }^{3}$ Nous empruntons cette formule à Laurent Thévenot (1997).
}

des études de marché effectuées - seraient plus enclins à acheter des fruits vendus sous l'appellation «châtaigne » (Bousigon, 1999). Avançons une explication.

\section{Retour vers le local : la châtaigne, produit naturel et sain}

La disjonction nature/culture est aujourd'hui retravaillée dans l'espace social sous la forme d'un renversement de points de vue opéré au bénéfice de la grandeur naturelle, maintenant célébrée, des modes de production et des produits agroalimentaires. Le souci pour l'écologie et pour le passé, que Marc Guillaume identifie comme des valeurs refuge des sociétés modernes (Guillaume, 1978) et que, $\mathrm{d}^{\prime}$ une certaine manière, les produits traditionnels supportent de façon convergente, oriente de façon singulière l'acte d'achat et de consommation. Après des siècles d'un arrachement à la nature à travers des systèmes agricoles reposant sur la maîtrise technique des ressources naturelles, après la quête de produits normalisés et standardisés qui ont un temps incarné la modernité alimentaire, on assiste aujourd'hui au réenchantement de cette proximité avec la nature. Ce mouvement converge avec un net attrait pour le local, la typicité et la singularité que le phénomène de mondialisation tend à conforter.

\section{Protection de l'environnement et castanéiculture traditionnelle}

L'argument écologique est présent dès le XVIII ${ }^{\mathrm{e}}$ siècle dans la littérature savante ou de vulgarisation. Parmi les premiers arguments, on repère des motifs d'ordre hygiéniste. Ils font du châtaignier un arbre de salubrité public, comme le soulignait alors Duhamel du Monceau (1764) : «Autrefois, de grandes forêts de cet arbre attiraient sur le sol de la France ces nuages salutaires, ces vapeurs bienfaisantes, qui adoucissent la température et fertilisent la terre. [... ] Leur beau et large feuillage, après avoir purifié et rafraîchi l'air pendant l'été, engraisse et fertilise la terre pendant l'hiver. »

Ce registre est encore fort au début du XXe siècle, d'autant que la castanéiculture (comme bien d'autres productions végétales françaises) traverse une crise particulièrement grave. Henri Blin, publiciste agricole de son métier, défend la cause du châtaignier en avançant que «sa ramure puissante le rend précieux pour constituer un épais rideau contre les miasmes et les grands vents » (Blin, 1904). À la même époque, Jean-Baptiste Lavialle décrit à son tour le châtaignier comme l'une "des essences forestières les plus utiles $[. .$.$] pour la bonne in-$ fluence qu'il exerce sur l'assainissement du climat, sur le régime des eaux, sur la température du sol, sur l'action des vents et, par suite, sur la fécondité même des 
terres environnantes » (Lavialle, 1906). Que sont devenus ces arguments, plus de deux siècles après Duhamel du Monceau ? L'hygiénisme a fait place à un registre écologique et environnemental qui ne concerne plus la seule essence Castanea sativa, mais s'étend aussi à la manière dont l'arbre est cultivé et entretenu. Autrement dit, le lien est explicitement fait entre l'espèce (le châtaignier européen), l'espace (la châtaigneraie) et les pratiques culturales (la castanéiculture). Celles-ci apparaissent, en effet, bien singulières dans l'économie capitaliste du secteur agricole.

Pour mieux comprendre la mobilisation contemporaine des arguments écologiques, il faut apporter quelques précisions sur les variétés hybrides interspécifiques évoquées plus haut. Ces fruits sont produits depuis une vingtaine d'années, y compris en Ardèche, selon les méthodes classiques de l'arboriculture intensive (densité et rendements importants, irrigation, soins constants, terrains plats et mécanisables, etc.). Par comparaison, les châtaigneraies des pentes, où la mécanisation n'est pas concevable, sont de bien moindre rapport étant donné le grand âge des arbres et leur faible densité à l'hectare (une quarantaine à l'hectare contre deux cents en verger intensif); il n'y a pas d'irrigation, pas d'engrais de synthèse ni de pesticides et la plupart des interventions sur les arbres sont indirectes, à l'exception de l'élagage et des soins prophylactiques. Il s'ensuit un rapport technique très particulier à l'arbre, à la châtaigneraie, aux ressources naturelles et au temps (Dupré, 2003). Ce rapport a toujours été analysé de façon ambivalente. D'un côté, la facilité technique avec laquelle s'obtiennent les châtaignes a été et demeure condamnée ; la production est si peu interventionniste, relativement aux modèles arboricoles intensifs, qu'elle a été reléguée au rang de simple cueillette. De l'autre, des arguments presque identiques servent - mais c'est là chose récente - à mettre en avant une agriculture respectueuse de l'environnement et préservatrice de la biodiversité.

En effet, la châtaigneraie est un agroécosystème remarquable ${ }^{4}$ à plusieurs titres, comme l'avaient déjà souligné, à leur manière et il y a plusieurs siècles, les auteurs cités ci-dessus. Elle constitue tout d'abord un espace agricole productif naturel qui, non seulement préserve, mais héberge une biodiversité sauvage (fleurs, insectes, oiseaux, champignons, graminées, fruits) et domestique importante (plusieurs variétés de châtaignes par parcelle). Ensuite, sa fonctionnalité écologique est reconnue : une châtaigneraie entretenue lutte efficacement contre l'érosion et prévient les incendies dans ces régions méditerranéennes exposées aux sécheresse et aux pluies diluviennes.

\footnotetext{
${ }^{4}$ Il correspond d'ailleurs à l'habitat naturel 9260 répertorié dans les cahiers d'habitats élaborés dans le cadre de la directive Habitats (réseau Natura 2000). On y parle de "forêt de châtaigniers ».
}

La dernière réforme de la PAC, mais également l'ensemble des politiques publiques de l'espace rural, vont dans le sens d'une agriculture plus respectueuse de l'environnement. Dans un tel contexte, les systèmes et les espaces de culture castanéicoles connaissent une certaine requalification. Toutefois, celle-ci ne suffit pas à lever l'ambiguïté d'une production sur laquelle pèse encore un "soupçon d'archaïsme" (Rumpala, 1998). En effet, d'un côté, l'écolabellisation atteste son caractère agricole et l'arrache donc à son statut de ressource naturelle spontanée. Tenue à l'écart des excès industriels, la production de châtaignes offrirait aujourd'hui un exemple presque expérimental de bonne entente socio-technique et de commerce équitable avec la nature. D'un autre côté, une telle distinction ne résout pas le problème de la valorisation des fruits, dont la production est confrontée à d'énormes difficultés. Prenons l'exemple de la diversité variétale reconnue.

\section{Valorisation marchande et diversité variétale}

La diversité variétale est une des caractéristiques majeures des systèmes de production paysans. Elle permettait d'abord de s'adapter à une large palette de conditions pédologiques et climatiques. Par ailleurs, les récoltes s'échelonnant en fonction de la maturité des variétés et de leur résistance variable aux maladies et aux incidents climatiques, elle mettait les paysans à l'abri d'une récolte nulle. Enfin, chaque variété était utilisée d'une façon singulière. Le châtaignier étant quant à lui autostérile, la présence de plusieurs variétés s'impose : l'arbre a besoin du pollen d'une autre variété pour pouvoir fructifier. Longtemps décriée ( «les variétés produites en Ardèche sont nombreuses, trop nombreuses », rappelait un expert dans les années 1930), cette diversité biologique cultivée est réhabilitée depuis les années 1980. La création de l'association Les Croqueurs de Pommes et l'organisation d'un colloque national autour de cette question ${ }^{5}$ marquent la montée en puissance d'un intérêt du public (qui ne se réduit plus alors à la seule classe des consommateurs) pour cette cause. Ce phénomène conduit peu à peu à l'émergence d'un engouement pour les espèces fruitières locales anciennes ou disparues, oubliées ou paysannes ${ }^{6}$. Mais, pour autant qu'elle mobilise le public, la valorisation marchande de cette biodiversité ne va pas sans difficultés.

En effet, une partie de la commercialisation est assurée par des coopératives et des metteurs en marché

\footnotetext{
${ }^{5}$ Colloque organisé par le Bureau des ressources génétiques (BRG) et l'Association française pour la conservation des espèces végétales (AFCEV) à Nancy en 1984.

6 Dans l'édition de 1986 du Catalogue officiel des espèces et variétés de fruits et de légumes, le Comité technique permanent de la sélection des plantes cultivées (CTPS) ouvre ses listes aux variétés d'amateurs, également dites d'intérêt régional.
} 
privés. L'agréage répartit les fruits en plusieurs catégories marchandes homogènes qui ne tiennent nullement compte de l'ensemble de la palette variétale. La singularité morphologique de l'une d'elles peut rendre difficile l'épreuve de calibrage et obliger à un traitement distinct, alors que son seul tonnage ne le justifierait pas; c'est le cas pour la variété ardéchoise Aguyane, dont la forme triangulaire et plate pose problème. Ses fruits ne passent pas dans les grilles classiques prévues pour chacun des trois groupes de calibre. Leur commercialisation suppose donc une opération supplémentaire encore consentie par les coopérateurs étant donné son tonnage significatif. En revanche, une autre variété atypique moins récoltée ne sera sans doute pas l'objet d'une telle attention. Trois catégories de fruits sont clairement identifiées.

La première rassemble les variétés hybrides interspécifiques «à caractère marron ». Généralement précoces, ces fruits ouvrent la saison à des cours pouvant atteindre 2,6 euros le kilo. Ils présentent les caractéristiques du superlatif marron industriel qui en a inspiré la création : gros calibre, pas de cloisonnement, bonne tenue à la cuisson, aptitude à l'épluchage mécanique. Il s'agit de fruits hors normes : très, voire trop gros ; très, voire trop précoces; chers, voire trop chers. Dans les discours locaux, les hybrides souffrent $d$ 'un excès de culture et deviennent suspects aux yeux des consommateurs avertis ${ }^{7}$. On dit d'eux qu'ils « ont le coup d'œil » mais le « goût de rave", qu'ils sont «trafiqués » et "artificiels » relativement à leur statut socio-technique d'hybrides et à leur méthode de production qui tranche avec les pratiques traditionnelles (Dupré, 2003). Viennent ensuite des variétés locales nobles, celles-là mêmes qui ont été évincées de l'épreuve industrielle au début du XXe siècle. Elles sont vendues selon leur calibre (mais aussi selon les commandes et parfois les stocks d'étiquettes disponibles !), tantôt sous l'appellation «châtaignes ", tantôt sous l'appellation "marrons ». La spécificité variétale est en revanche toujours affichée. Il s'agit des variétés Bouche-Rouge, de la Comballe et de la Merle, qui sont reconnues comme des fruits de bouche de grande qualité. Enfin, viennent les variétés d'industrie complètement dépréciées en raison de leur très petit calibre et bien que certaines $\mathrm{d}$ 'entre elles, comme la Pourette, soient reconnues pour leur très grande qualité gustative. Elles sont vendues aujourd'hui aux industriels qui en font, nous l'avons vu, de la crème de... marrons. Leur très faible valorisation économique et culturelle (en moyenne 0,30 euros le kilo) et l'énorme travail de récolte qu'elles exigent dissuadent les producteurs de continuer l'entretien de ces châtaigniers.

Si la manière de catégoriser la diversité variétale castanéicole présente depuis plusieurs siècles une certaine constance, un renversement s'est toutefois opéré.

\footnotetext{
7 Sans entrer dans les détails, l'affaire est bien évidemment autrement plus complexe (Dupré, 2003).
}

La valorisation du terme "châtaigne", sensible jusque dans le choix très discuté du nom d'un projet d'AOC en cours, en constitue l'un des indices. D'un côté, on peut y voir la valorisation du lien entre l'arbre, le fruit, des espaces de production singuliers et la valeur agrienvironnementale de pratiques proches de l'agroforesterie. Et c'est bien dans cette perspective que se sont engagées plusieurs opérations de patrimonialisation et de labellisation conjointes de l'Ardèche, de ses paysages de châtaigneraies accueillantes et apaisantes, du fruit et du châtaignier (Dupré, 2004). Ce lien, qu'il fallait autrefois masquer, est donc, depuis peu, valorisé; il est le gage d'une qualité particulière qui contribue à faire de la châtaigne un produit rassurant et, donc, à inspirer confiance au consommateur. De l'autre, et dans une continuité évidente, la requalification de l'acte de consommation de ces fruits historiquement associés à une nourriture grossière et trop nourrissante occupe une place importante dans la relance de la filière (Bruneton-Governatori, 1984).

\section{Authenticité et qualité nutritionnelle}

L'objectif de productivité des années 1970 s'est déplacé vers une exigence de sécurité sanitaire des biens agroalimentaires que les récentes crises du secteur n'ont fait qu'accentuer. La filière châtaigne n'est pas, on s'en doute, particulièrement visée par les mêmes risques que ceux de la filière bovine, par exemple. En revanche, elle se heurte à deux limites majeures : c'est un produit saisonnier dont la consommation reste très marginale et il est réputé trop nourrissant. La diversification de la gamme de produits à base de châtaigne et la réhabilitation de ses qualités nutritionnelles constituent deux façons originales d'anticiper ces fragilités.

La première manière de déjouer ces fragilités vise à démultiplier les territoires de consommation en exploitant les statuts du fruit : substitut de céréales, fruit frais ou légume d'accompagnement, dessert, encas, etc. (Rio, 1986). Les formes anciennes de conservation, aujourd'hui qualifiées promptement $d^{\prime}$ " authentiques », sont remises au goût du jour, avec des succès mitigés selon les cas. Les châtaignes sèches (réhydratées avant d'être consommées), archétypes de la consommation vivrière, ainsi que la farine de châtaigne en sont l'illustration parfaite. Ces produits historiques, que l'évolution de l'alimentation et des techniques de conservation ont conduit à délaisser, invitent le consommateur à plonger dans un ailleurs historique, géographique et technique jouant de cette proximité avec la nature. Ces produits permettent de bien valoriser les excellentes mais petites variétés, qui autrement sont bradées aux industries. Le texte suivant, figurant sur un emballage de farine de châtaigne, en est une illustration: «Nos châtaignes ramassées dès octobre sont séchées 
au feu de bois dans la clède ${ }^{8}$. Lentement, elles sèchent pendant 20 à 30 jours. Nous les débarrassons de leurs deux peaux en les passant dans la machine à piser. Une fois triées à la main, nous les portons au moulin où elles donneront cette farine. Produit naturel, sans additif, ni conservateur. . . » La référence à la lenteur des opérations, à la manipulation manuelle, au soin apporté aux fruits et à leur caractère naturel confirme l'origine fermière du produit et déploie tout un univers local, aujourd'hui fortement valorisé. Par ailleurs, la composition chimique de la farine est détaillée; elle installe le discours dans le registre nutritionnel et affiche une impressionnante diversité non seulement en glucides, mais surtout en vitamines (C, PP, B1, B5, B2) et en sels minéraux (calcium, phosphore, fer, potassium, zinc, magnésium, cuivre, etc.). Ces informations, que l'on commence à trouver couramment sur les emballages et les prospectus, s'appuient sur deux nouveaux types de caution : la qualité nutritionnelle des aliments $^{9}$ et leur grandeur naturelle et artisanale comme gages de leur qualité.

L'idée d'une nourriture de substitution est ici neutralisée par la mise en exergue du caractère festif, convivial et simple du fruit. La châtaigne est alors définie relativement au monde domestique (fabrication artisanale, personnification et singularisation du produit, localisation dans un espace précis et garantie de son origine). Les consommateurs ciblés par les campagnes promotionnelles sont les jeunes enfants, les sportifs et les personnes âgées. Un tel panel atteste du caractère transgénérationnel de la consommation de châtaignes et compose les repères d'un cadre sécurisant parce que précisément familial. Mais l'acte de consommation ne se réduit pas à l'ingestion de l'aliment : il se double d'une incorporation des propriétés symboliques de ce dernier et repose largement sur l'évocation sensible des paysages de châtaigneraie. On est loin du dédain autrefois affiché pour ce fruit qui apparaissait d'autant plus dévalorisé que sa consommation était locale. La caution nutritionnelle rassure le consommateur, tandis que la seconde, domestique, naturelle et paysagère, en fait un "idiot du voyage ", selon l'expression de J.-D. Urbain (Urbain, 1991). La singularité du produit se reporte sur le consommateur, à son tour singulier.

À l'opposé d'un espace intermédiaire et dangereux, la châtaigneraie devient alors un lieu non seulement

\footnotetext{
${ }^{8}$ La clède est un petit bâtiment en pierre dans lequel un foyer (souvent remplacé aujourd'hui par un poêle) est entretenu durant une quarantaine de jours pour faire sécher les fruits. Ces derniers sont déposés sur un plancher ajouré au-dessus du feu.

${ }^{9}$ Ce type d'argumentation n'est toutefois pas nouveau. Au XIX ${ }^{e}$ siècle, lorsque Augustin Parmentier s'est intéressé à la châtaigne, c'était bien, comme il l'avait fait avec la pomme de terre, dans l'idée de résoudre les problèmes d'alimentation des populations rurales, en insistant donc sur le caractère sain et nutritif du fruit (Parmentier, 1780).
}

productif, mais aussi récréatif et ouvert aux touristes. Mais si la plus-value culturelle est réelle, la valorisation économique reste, quant à elle, faible en dehors des stratégies individuelles de certains producteurs. On peut y voir deux raisons. Premièrement, parce que certains produits sont confrontés à des échecs : le pain à la châtaigne, par exemple, ne fait pas recette et l'invention d'une boisson énergétique lactée à la châtaigne a connu beaucoup de déboires. Deuxièmement, parce que cette diversification soutenue par ce nouveau registre d'argumentation ne résout pas pour autant les grosses difficultés de la filière : problèmes phytosanitaires, récolte très pénible, prix dérisoire des fruits, problèmes de vols de récolte en châtaigneraie, faible productivité à l'hectare.

Pour finir, on peut se demander comment les exigences en termes de diversité bioculturelle, de contrôle de l'origine et de qualité nutritionnelle trouveront à se reporter, non plus sur la seule châtaigne et son histoire, mais également, et de façon explicite, sur l'espace de nature qu'elle convoque et dont elle est à la fois le support et le produit. Autrement dit, ces trois préoccupations s'inscriront-elles un jour dans un acte de consommation militant qui verrait s'étendre le souci de protection de son propre corps à celui de l'environnement? Peut-on imaginer, un jour, trouver une invitation qui dirait : « Mangez des châtaignes ardéchoises, vous lutterez contre l'érosion des pentes, contribuerez à la lutte contre les incendies et protégerez un habitat naturel reconnu par l'Union européenne »? Et qui, cela va de soi, supposerait aussi que les hommes et les femmes qui produisent ces châtaignes puissent en tirer effectivement un revenu agricole.

\section{Références}

Bessy, C., Chateauraynaud, F., 1995. Experts et faussaires : pour une sociologie de la perception, Paris, Métailié.

Blin, H., 1904. Manuel pratique de la culture, de l'exploitation et des utilisations du châtaignier, contenant : étude botanique, industrie forestière et chimique, Paris, L. Mulo.

Bousigon, J.-C., 1999. Le marché de la châtaigne. Conférence prononcée au Salon international des fruits et des légumes, Agen, Orgagri-SIFEL.

Bruneton-Governatori, A., 1984. Le Pain de bois : ethnohistoire de la châtaigne et du châtaignier, Toulouse, Éché Éditeur.

Clerc, L, 1828 ( $2^{\mathrm{e}}$ éd.). Manuel de l'amateur de marrons et de châtaignes ou l'Art de cultiver le châtaignier, de le multiplier, d'en récolter le fruit favorablement, de le conserver d'une année à l'autre sans qu'il perde rien de sa bonté ordinaire, et de le préparer de la manière la plus agréable, par un grand nombre de méthodes usitées, soit en France, soit dans les pays étrangers. Suivi des propriétés alimentaires et médicales de ce fruit, Paris, Librairie française et étrangère.

Commission du châtaignier, 1951. Semaine internationale $d u$ châtaignier, septembre 1950, Nancy, École nationale des eaux et forêts.

Demolins, E., 1900, Les Français d'aujourd'hui : les types sociaux $d u$ midi de la France, Paris, Firmin Didot et $C^{i e}$. 
Duhamel du Monceau, H.-L., 1764. De l'exploitation des bois, ou Moyens de tirer un parti avantageux des taillis, demi-futaies et hautes futaies, et d'en faire une juste estimation; avec la Description des arts qui se pratiquent dans les forêts : faisant partie du Traité complet du bois et des forests, Paris, H.-L. Guérin et L.-F. Delatour.

Dupré, L., 2002. Du marron à la châtaigne d'Ardèche : la relance d'un produit régional, Paris, CTHS.

Dupré, L., 2003. Traiter le châtaignier-en-personne : pour revisiter Haudricourt, in Lieutaghi, P., Musset, D., Reckinger, R. (Eds), Plantes, sociétés, savoirs, symboles : matériaux pour une ethnobotanique européenne, Actes du séminaire d'ethnobotanique de Salagon, Forcalquier, Les Alpes de lumière / Mane, Salagon, Musée-conservatoire ethnologique de HauteProvence, 121-128.

Dupré, L., 2004. De la châtaigne au châtaignier : de la disqualification à la requalification de la production castanéicole en Ardèche, in Charlery de la Masselière B. (Eds), Fruits du terroir, fruits défendus : identités, mémoires et territoires, Toulouse, Presses universitaires du Mirail, 105-115.

Faugier, C., 1935. L'industrie des Marrons de confiserie, Congrès méditerranéen du châtaignier, Aubenas, 25-27 octobre 1935, Privas, Imprimerie Volle, 218-224.

Guillaume M., 1978. Éloge du désordre, Paris, Le Seuil.

Larousse, P., 1866-1879. Grand dictionnaire universel du XIX ${ }^{\mathrm{e}}$ siècle, Génève/Paris, Slatkine, reprint 1982.
Lavialle, J.-B., 1906. Études agronomiques et sociales. Le Châtaignier, sa culture, utilisation de ses produits, ses maladies et leurs remèdes, conclusions pratiques, Paris, Vigot Frères.

Marchenay, P., 1981. Ethnobotanique et conservation génétique. L'exemple des arbres fruitiers, Journal d'agriculture traditionnelle et de botanique appliquée, XXVIII, 2, 85-158.

Parmentier, A., 1780. Traité de la châtaigne, Paris/Bastia, Monory. Reyne, J., 1995 (1 $1^{\mathrm{re}}$ éd. 1984). Marrons et châtaignes d'Ardèche, Privas, SPCMA.

Rio, Y., 1986. Diversité des produits et alimentation humaine, in La Diversité des plantes légumières, hier, aujourd'hui et demain, Actes du symposium organisé à Angers du 17 au 19 octobre 1985 par l'Association pour l'inventaire et la conservation des plantes cultivées dans les Pays de Loire, l'Association des conservatoires français d'espèces végétales et le Bureau des ressources génétiques, Paris, Journal d'agriculture traditionnelle et de botanique appliquée, diffusion Tec \& Doc, 169-174.

Rumpala, Y., 1998. Les ambiguïtés d'une intervention publique dans la préservation des paysages : retour sur les labels "paysages de reconquête », Natures Sciences Sociétés, 6, 3, 39-44.

Thévenot, L., 1997. Un gouvernement par les normes. Pratiques et politiques des formats d'information, Raisons pratiques, 8 , 205-242.

Urbain, J.-D., 1991. L'Idiot du voyage : histoires de touristes, Paris, Plon.

Reçu le 2 mars 2005. Accepté le 8 septembre 2005.

To access this journal online: www.edpsciences.org 